\title{
Proof Complexity of Propositional Default Logic ${ }^{\star}$
}

\author{
Olaf Beyersdorff ${ }^{1}$, Arne Meier ${ }^{2}$, Sebastian Müller ${ }^{3}$, Michael Thomas², and \\ Heribert Vollmer ${ }^{2}$ \\ 1 Institute of Computer Science, Humboldt University Berlin, Germany \\ beyersdo@informatik.hu-berlin.de \\ 2 Institute of Theoretical Computer Science, Leibniz University Hanover, Germany \\ \{meier, thomas, vollmer\}@thi.uni-hannover.de \\ 3 Faculty of Mathematics and Physics, Charles University Prague, Czech Republic \\ smueller@informatik.hu-berlin.de
}

\begin{abstract}
Default logic is one of the most popular and successful formalisms for non-monotonic reasoning. In 2002, Bonatti and Olivetti introduced several sequent calculi for credulous and skeptical reasoning in propositional default logic. In this paper we examine these calculi from a proof-complexity perspective. In particular, we show that the calculus for credulous reasoning obeys almost the same bounds on the proof size as Gentzen's system $L K$. Hence proving lower bounds for credulous reasoning will be as hard as proving lower bounds for $L K$. On the other hand, we show an exponential lower bound to the proof size in Bonatti and Olivetti's enhanced calculus for skeptical default reasoning.
\end{abstract}

\section{Introduction}

Trying to understand the nature of human reasoning has been one of the most fascinating adventures since ancient times. It has long been argued that due to its monotonicity, classical logic is not adequate to express the flexibility of commonsense reasoning. To overcome this deficiency, a number of formalisms have been introduced (cf. [20]), of which Reiter's default logic [21] is one of the most popular and widely used systems. Default logic extends the usual logical (firstorder or propositional) derivations by patterns for default assumptions. These are of the form "in the absence of contrary information, assume ...". Reiter argued that his logic adequately formalizes human reasoning under the closed world assumption. Today default logic is widely used in artificial intelligence and computational logic.

The semantics and the complexity of default logic have been intensively studied during the last decades (cf. [7] for a survey). In particular, Gottlob [13] has identified and studied two reasoning tasks for propositional default logic: the credulous and the skeptical reasoning problem which can be understood as analogues of the classical problems SAT and TAUT. Due to the stronger expressibility of default logic, however, credulous and skeptical reasoning become harder than their classical counterparts - they are complete for the second level $\Sigma_{2}^{\mathrm{p}}$ and $\Pi_{2}^{\mathrm{p}}$ of the polynomial hierarchy, respectively [13].

\footnotetext{
* Research supported in part by DFG grants KO 1053/5-2 and VO 630/6-1, by a grant from the John Templeton Foundation, and by the Marie Curie FP7 Initial Training Network MALOA (no. 238381).
} 
Less is known about the complexity of proofs in default logic. While there is a rich body of results for propositional proof systems (cf. [17]), proof complexity of non-classical logics has only recently attracted more attention, and a number of exciting results have been obtained for modal and intuitionistic logics [14-16]. Starting with Reiter's work [21], several proof-theoretic methods have been developed for default logic (cf. $[1,11,18,19,22]$ and [9] for a survey). However, most of these formalisms employ external constraints to model nonmonotonic deduction and thus cannot be considered purely axiomatic (cf. [10] for an argument). This was achieved by Bonatti and Olivetti [4] who designed simple and elegant sequent calculi for credulous and skeptical default reasoning. Subsequently, Egly and Tompits [10] extended Bonatti and Olivetti's calculi to first-order default logic and showed a speed-up of these calculi over classical first-order logic, i.e., they construct sequences of first-order formulae which need long classical proofs but have short derivations using default rules.

In the present paper we investigate the original calculi of Bonatti and Olivetti [4] from a proof-complexity perspective. Apart from some preliminary observations in [4], this comprises, to our knowledge, the first comprehensive study of lengths of proofs in propositional default logic. Our results can be summarized as follows. Bonatti and Olivetti's credulous default calculus $B O_{\text {cred }}$ obeys almost the same bounds to the proof size as Gentzen's propositional sequent calculus $L K$, i.e., we show that upper bounds to the proof size in both calculi are polynomially related. The same result also holds for the proof length (the number of steps in the system). Thus, proving lower bounds to the size of $B O_{\text {cred }}$ will be as hard as proving lower bounds to $L K$ (or, equivalently, to Frege systems), which constitutes a major challenge in propositional proof complexity $[5,17]$. This result also has implications for automated theorem proving. Namely, we transfer the non-automatizability result of Bonet, Pitassi, and Raz [6] for Frege systems to default logic: $B O_{\text {cred }}$-proofs cannot be efficiently generated, unless factoring integers is possible in polynomial time.

While already $B O_{\text {cred }}$ appears to be a strong proof system for credulous default reasoning, admitting very concise proofs, we also exhibit a general method of how to construct a proof system $\operatorname{Cred}(P)$ for credulous reasoning from a propositional proof system $P$. This system $\operatorname{Cred}(P)$ bears the same relation to $P$ with respect to proof size as $B O_{\text {cred }}$ does to $L K$. Thus, choosing for example $P$ as extended Frege might lead to stronger proof systems for credulous reasoning.

For skeptical reasoning, the situation is different. Bonatti and Olivetti [4] construct two proof systems for this task. While they already show an exponential lower bound for their first skeptical calculus, we obtain also an exponential lower bound to the proof length in their enhanced skeptical calculus. This lower bound also holds if the enhanced calculus is augmented by further rules such as the cut rule.

This paper is organized as follows. In Sect. 2 we start with some background information on proof systems and default logic. The calculi of Bonatti and Olivetti [4] consist of four main ingredients: classical sequents, antisequents to refute non-tautologies, a residual calculus, and default rules. Thus we start our investigation in Sect. 3 by analyzing the preliminary antisequent and residual 
calculi. Our main results on the proof complexity of credulous and skeptical default reasoning follow in Sects. 4 and 5, respectively. In Sect. 6, we conclude with a discussion and some open questions.

\section{Preliminaries}

We assume familiarity with propositional logic and basic notions from complexity theory (cf. [17]). By $\mathcal{L}$ we denote the set of all propositional formulae over some fixed standard set of connectives. For $T \subseteq \mathcal{L}$, the set of all logical consequences of $T$ will be denoted by $T h(T)$.

\subsection{Proof Systems}

Cook and Reckhow [8] defined the notion of a proof system for an arbitrary language $L$ as a polynomial-time computable function $f$ with range $L$. A string $w$ with $f(w)=x$ is called an $f$-proof for $x \in L$. Proof systems for $L=$ TAUT are called propositional proof systems. The sequent calculus $L K$ of Gentzen [12] is one of the most important and best studied propositional proof systems. It is well known that $L K$ and Frege systems mutually p-simulate each other(cf. [17]).

There are two measures which are of primary interest in proof complexity. The first is the minimal size of an $f$-proof for some given element $x \in L$. To make this precise, let $s_{f}(x)=\min \{|w| \mid f(w)=x\}$ and $s_{f}(n)=\max \left\{s_{f}(x) \mid\right.$ $|x| \leq n\}$. We say that the proof system $f$ is $t$-bounded if $s_{f}(n) \leq t(n)$ for all $n \in \mathbb{N}$. If $t$ is a polynomial, then $f$ is called polynomially bounded. Another interesting parameter of a proof is the length defined as the number of proof steps. This measure only makes sense for proof systems where proofs consist of lines containing formulae or sequents. This is the case for $L K$ and most systems studied in this paper. For such a system $f$, we let $t_{f}(\varphi)=\min \{k \mid$ $f(\pi)=\varphi$ and $\pi$ uses $k$ steps $\}$ and $t_{f}(n)=\max \left\{t_{f}(\varphi)|| \varphi \mid \leq n\right\}$. Obviously, it holds that $t_{f}(n) \leq s_{f}(n)$, but the two measures are even polynomially related for a number of natural systems as extended Frege (cf. [17]).

For sequent calculi one distinguishes between dag-like and tree-like proofs where in the latter notion each derived sequent can be used at most once as a prerequisite of a rule. While for $L K$ these two measures are equivalent [17], we will concentrate here only on the stronger dag-like model.

\subsection{Default Logic}

Default logic is an extension of classical logic that has been proposed by Reiter [21]. The logic is non-monotonic in the sense that an increase in information may decrease the number of consequences. A default theory $\langle W, D\rangle$ consists of a set $W$ of propositional sentences and a set $D$ of defaults. A default (rule) $\delta$ is an inference rule of the form $\frac{\alpha: \beta}{\gamma}$, where $\alpha$ and $\gamma$ are propositional formulae and $\beta$ is a set of propositional formulae. The prerequisite $\alpha$ is also referred to as $p(\delta)$, the formulae in $\beta$ are called justifications (referred to as $j(\delta)$ ), and $\gamma$ is the conclusion that is referred to as $c(\delta)$. Stable extensions are originally defined in 
terms of a fixed-point equation [21], but we use the following characterization as a starting definition:

Theorem 1 (Reiter [21]). Let $E \subseteq \mathcal{L}$ be a set of formulae and $\langle W, D\rangle$ be a default theory. Furthermore let $E_{0}=W$, and

$$
E_{i+1}=T h\left(E_{i}\right) \cup\left\{c(\delta) \mid \delta \in D, E_{i} \vdash p(\delta), \neg j(\delta) \cap E=\emptyset\right\},
$$

where $\neg j(\delta)$ denotes the set of all negated sentences contained in $j(\delta)$. Then $E$ is a (stable) extension of $\langle W, D\rangle$ if and only if $E=\bigcup_{i \in \mathbb{N}} E_{i}$.

A default theory $\langle W, D\rangle$ can have none or several stable extensions (cf. [2,13] for examples). A sentence $\psi \in \mathcal{L}$ is credulously entailed by $\langle W, D\rangle$ if $\psi$ holds in some stable extension of $\langle W, D\rangle$. If $\psi$ holds in every extension of $\langle W, D\rangle$, then $\psi$ is skeptically entailed by $\langle W, D\rangle$.

Default rules with empty justification are called residues. Let $\mathcal{L}^{\text {res }}=\mathcal{L} \cup$ $\left\{\frac{\alpha}{\gamma} \mid \alpha, \gamma \in \mathcal{L}\right\}$ be the set of all formulae and residues. Residues can be used to alternatively characterize stable extensions. For a set $D$ of defaults and $E \subseteq \mathcal{L}$ let

$$
R E S(D, E)=\left\{\frac{p(\delta)}{c(\delta)} \mid \delta \in D, E \cap \neg j(\delta)=\emptyset\right\} .
$$

Apparently, $R E S(D, E)$ is a set of residues. We can then build stable extensions via the following closure operator. For a set $R$ of residues we define $C l_{0}(W, R)=$ $W$ and

$$
C l_{i+1}(W, R)=T h\left(C l_{i}(W, R)\right) \cup\left\{\gamma \mid \frac{\alpha}{\gamma} \in R, \alpha \in T h\left(C l_{i}(W, R)\right)\right\} .
$$

Let $C l(W, R)=\bigcup_{i=0}^{\infty} C l_{i}(W, R)$. Then for the sets $E_{i}$ from Theorem 1 the following holds:

Proposition 2 (Bonatti, Olivetti [4]). Let $\langle W, D\rangle$ be a default theory and let $E \subseteq \mathcal{L}$. Then $E_{i}=C l_{i}(W, R E S(D, E))$ for all $i \in \mathbb{N}$. In particular, $E$ is a stable extension of $\langle W, D\rangle$ if and only if $E=C l(W, R E S(D, E))$.

If $D$ only contains residues, then there is an easier way of characterizing $C l$ :

Lemma 3 (Bonatti, Olivetti [4]). For $D \subseteq \mathcal{L}^{\text {res }} \backslash \mathcal{L}, W \subseteq \mathcal{L}$, and for $i \in \mathbb{N}$ let

$$
C_{0}=W \quad \text { and } \quad C_{i+1}=C_{i} \cup\left\{\gamma \mid \frac{\alpha}{\gamma} \in D, \alpha \in T h\left(C_{i}\right)\right\} .
$$

Then $\gamma \in C l(W, D)$ if and only if there exists $k \in \mathbb{N}$ with $\gamma \in T h\left(C_{k}\right)$.

\section{Proof Complexity of the Antisequent and Residual Calculi}

Bonatti and Olivetti's calculi for default logic use four main ingredients: usual propositional sequents and rules of $L K$, antisequents to refute formulae, residual rules, and default rules. In this section we will investigate the complexity of the antisequent calculus $A C$ and the residual calculus $R C$. 
We start with the definition of Bonatti's antisequent calculus $A C$ from [3]. A related refutation calculus for first-order logic was previously developed by Tiomkin [23]. In $A C$ we use antisequents $\Gamma \nvdash \Delta$, where $\Gamma, \Delta \subseteq \mathcal{L}$. Intuitively, $\Gamma \nvdash \Delta$ means that $\bigvee \Delta$ does not follow from $\wedge \Gamma$. Axioms of $A C$ are all sequents $\Gamma \nvdash \Delta$, where $\Gamma$ and $\Delta$ are disjoint sets of propositional variables. The inference rules of $A C$ are shown in Fig. 1. For this calculus, Bonatti [3] shows:

$$
\begin{array}{ccc}
\frac{\Gamma \nvdash \Sigma, \alpha}{\Gamma, \neg \alpha \nvdash \Sigma}(\neg \nvdash) & \frac{\Gamma, \alpha \nvdash \Sigma}{\Gamma \nvdash \Sigma, \neg \alpha}(\nvdash \neg) \\
\frac{\Gamma, \alpha, \beta \nvdash \Sigma}{\Gamma, \alpha \wedge \beta \nvdash \Sigma}(\wedge \nvdash) & \frac{\Gamma \nvdash \Sigma, \alpha}{\Gamma \nvdash \Sigma, \alpha \wedge \beta}(\nvdash \bullet \wedge) & \frac{\Gamma \nvdash \Sigma, \beta}{\Gamma \nvdash \Sigma, \alpha \wedge \beta}(\nvdash \wedge \bullet) \\
\frac{\Gamma \nvdash \Sigma, \alpha, \beta}{\Gamma \nvdash \Sigma, \alpha \vee \beta}(\nvdash \vee) & \frac{\Gamma, \alpha \nvdash \Sigma}{\Gamma, \alpha \vee \beta \nvdash \Sigma}(\bullet \vee \nvdash) & \frac{\Gamma, \beta \nvdash \Sigma}{\Gamma, \alpha \vee \beta \nvdash \Sigma}(\vee \bullet \nvdash) \\
\frac{\Gamma, \alpha \nvdash \Sigma, \beta}{\Gamma \nvdash \Sigma, \alpha \rightarrow \beta}(\nvdash \rightarrow) & \frac{\Gamma \nvdash \Sigma, \alpha}{\Gamma, \alpha \rightarrow \beta \nvdash \Sigma}(\bullet \rightarrow \nvdash) & \frac{\Gamma, \beta \nvdash \Sigma}{\Gamma, \alpha \rightarrow \beta \nvdash \Sigma}(\rightarrow \bullet \nvdash)
\end{array}
$$

Fig. 1. Inference rules of the antisequent calculus $A C$.

Theorem 4 (Bonatti [3]). The antisequent calculus $A C$ is sound and complete.

Concerning the size of proofs in the antisequent calculus we observe:

Proposition 5. The antisequent calculus $A C$ is polynomially bounded.

Proof. Observe that the calculus contains only unary inference rules, each of which reduces the logical complexity of one of the contained formulae (if perceived bottom-up). Thus each use of an inference rule decrements the size of the formulae by at least one. After a linear number of steps we end up with only propositional variables which we cannot reduce any further. Each antisequent is of linear size, hence the complete derivation has quadratic size.

The above observation is not very astounding, since, to verify $\Gamma \nvdash \Delta$ we could alternatively guess assignments to the propositional variables in $\Gamma$ and $\Delta$ and thereby verify antisequents in NP.

We now turn to the residual calculus $R C$ of Bonatti and Olivetti [4]. Its objects are residual sequents $\langle W, R\rangle \vdash \Delta$ and residual antisequents $\langle W, R\rangle \nvdash \Delta$ where $W, \Delta \subseteq \mathcal{L}$ and $R \subseteq \mathcal{L}^{r e s}$. The intuitive meaning is that $\Delta$ does (respectively does not) follow from $W$ using the residues $R$. The rules of $R C$ comprise of the inference rules from Fig. 2 together with the rules of $L K$ and $A C$. However, the use of rules from $L K$ and $A C$ is restricted to purely propositional (anti)sequents. For this calculus, Bonatti and Olivetti [4] showed:

Theorem 6 (Bonatti, Olivetti [4]). The residual calculus $R C$ is sound and complete, i.e., for all default theories $\langle W, R\rangle$ with $R \subseteq \mathcal{L}^{\text {res }}$ and all $\Delta \subseteq \mathcal{L}$, 


$$
\begin{array}{lr}
(\operatorname{Re} 1) \frac{\Gamma \vdash \Delta}{\Gamma, \frac{\alpha}{\gamma} \vdash \Delta} & (\operatorname{Re} 2) \frac{\Gamma \vdash \alpha}{\Gamma, \frac{\alpha}{\gamma} \vdash \Delta} \Gamma, \gamma \vdash \Delta \\
(\mathbf{R e} 3) \frac{\Gamma \nvdash \Delta \quad \Gamma \nvdash \alpha}{\Gamma, \frac{\alpha}{\gamma} \nvdash \Delta} & (\operatorname{Re} 4) \frac{\Gamma, \gamma \nvdash \Delta}{\Gamma, \frac{\alpha}{\gamma} \nvdash \Delta}
\end{array}
$$

Fig. 2. Inference rules of the residual calculus $R C$.

1. $\langle W, R\rangle \vdash \Delta$ is derivable in $R C$ if and only if $\bigvee \Delta \in C l(W, R)$;

2. $\langle W, R\rangle \nvdash \Delta$ is derivable in $R C$ if and only if $\bigvee \Delta \notin C l(W, R)$.

To bound the lengths of proofs in this calculus we exploit the property that residues only have to be used at a certain level and are not used to deduce any formulae afterwards (cf. Lemma 3). Using this we prove that the complexity of $R C$ is tightly linked to that of $L K$.

Lemma 7. There exist a polynomial $p$ and a constant $c$ such that $s_{R C}(n) \leq$ $p(n) \cdot s_{L K}(c n)$ and $t_{R C}(n) \leq p(n) \cdot t_{L K}(c n)$.

Proof. The proof consists of two parts. First we will show the bounds stated above for sequents. In the second part we will then show that antisequents even admit polynomial-size proofs in $R C$.

Assume first that we want to derive the sequent $\langle W, R\rangle \vdash \Delta$, where $W, \Delta \subseteq \mathcal{L}$ and $R=\left\{r_{1}, \ldots, r_{k}\right\}$ is a set of residues with $r_{i}=\frac{\alpha_{i}}{\gamma_{i}}$. Let $R^{\prime} \subseteq R$ be minimal with respect to the size $\left|R^{\prime}\right|$ such that $\left\langle W, R^{\prime}\right\rangle \vdash \Delta$. We may w.l.o.g. assume that $R^{\prime}=\left\{r_{1}, \ldots, r_{k^{\prime}}\right\}$ and $k^{\prime} \leq k$. Furthermore, by Lemma 3 , we may assume that the rules $r_{i}$ are ordered in the way they are applied when computing the sets $C_{i}$. In particular, this means that for each $i=1, \ldots, k^{\prime}$,

$$
W \cup\left\{\gamma_{1}, \ldots, \gamma_{i-1}\right\} \vdash \alpha_{i}
$$

is a true propositional sequent for which we fix an $L K$-proof $\Pi_{i}$. We augment $\Pi_{i}$ by $k^{\prime}-i$ applications of rule (Re1) to obtain

$$
\left\langle W \cup\left\{\gamma_{1}, \ldots, \gamma_{i-1}\right\},\left\{r_{i+1}, \ldots, r_{k^{\prime}}\right\}\right\rangle \vdash \alpha_{i} .
$$

Let us call the proof of this sequent $\Pi_{i}^{\prime}$.

The proof tree depicted in Fig. 3 for deriving $\langle W, R\rangle \vdash \Delta$ unfurls as follows. We start with an $L K$-proof for the sequent $W \cup\left\{\gamma_{1}, \ldots, \gamma_{k^{\prime}}\right\} \vdash \Delta$ and then apply $k^{\prime}$-times the rule $(\mathbf{R e} 2)$ in the step

$$
\frac{\left\langle W \cup\left\{\gamma_{1}, \ldots, \gamma_{i-1}\right\},\left\{r_{i+1}, \ldots, r_{k^{\prime}}\right\}\right\rangle \vdash \alpha_{i} \quad\left\langle W \cup\left\{\gamma_{1}, \ldots, \gamma_{i}\right\},\left\{r_{i+1}, \ldots, r_{k^{\prime}}\right\}\right\rangle \vdash \Delta}{\left\langle W \cup\left\{\gamma_{1}, \ldots, \gamma_{i-1}\right\},\left\{r_{i}, \ldots, r_{k^{\prime}}\right\}\right\rangle \vdash \Delta}
$$

to reach $\left\langle W, R^{\prime}\right\rangle \vdash \Delta$. To derive the left prerequisite we use the proof $\Pi_{i}^{\prime}$. Finally we use $k-k^{\prime}$ applications of the rule (Re1) to get $\langle W, R\rangle \vdash \Delta$.

Our proof for $\langle W, R\rangle \vdash \Delta$ uses at most $\left(k^{\prime}+1\right) \cdot t_{L K}(n)+\frac{k^{\prime}\left(k^{\prime}+1\right)}{2}+k$ steps, i.e., $t_{R C}(n) \leq \mathcal{O}\left(n \cdot t_{L K}(n)+n^{2}\right)$. Each sequent is of linear size. Hence, $s_{R C}(n) \leq p(n) \cdot s_{L K}(n)$ for some polynomial $p$. 


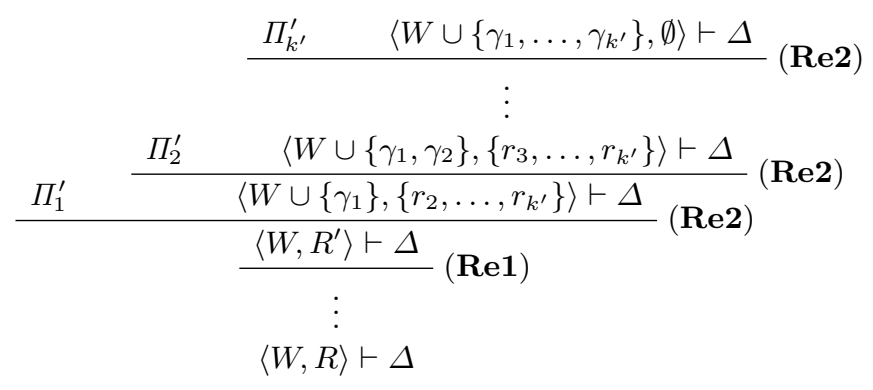

Fig. 3. Proof tree for the sequent $\langle W, R\rangle \vdash \Delta$ in the residual calculus.

In the second part of the proof we will now show that any true antisequent has an $R C$-proof of polynomial size, thus concluding the proof. Let $\langle W, R\rangle \nvdash \Delta$ be the antisequent we wish to prove. Again, let $R=\left\{r_{1}, \ldots, r_{k}\right\}$ with $r_{i}=\frac{\alpha_{i}}{\gamma_{i}}$, and let $\left\{i_{1}, \ldots, i_{\ell}\right\}=I \subseteq\{1, \ldots, k\}$ be a set of maximal cardinality such that $\left\langle W \cup \bigcup_{i \in I}\left\{\gamma_{i}\right\}\right\rangle \nvdash \Delta$ and let $I^{\prime}=\left\{i_{\ell+1}, \ldots, i_{k}\right\}=\{1, \ldots, k\} \backslash I$.

Because of $\langle W, R\rangle \nvdash \Delta$, the set $I$ contains all indices $i$ with $\alpha_{i} \in C l(W)$. Therefore, for each $j \in I^{\prime}$ we have $W \cup \bigcup_{i \in I}\left\{\gamma_{i}\right\} \nvdash \alpha_{j}$. We fix a polynomialsize $A C$-proof $\Pi_{j}$ of this antisequent. Augmenting these proofs with $\ell$ applications of $(\mathbf{R e} 4)$ we obtain a proof $\Pi_{j}^{\prime}$ of $\left\langle W, \bigcup_{i \in I}\left\{r_{i}\right\}\right\rangle \nvdash \alpha_{j}$. Similarly, as $\left\langle W \cup \bigcup_{i \in I}\left\{\gamma_{i}\right\}\right\rangle \nvdash \Delta$ we get a polynomial-size proof $\Pi_{k+1}^{\prime}$ of $\left\langle W, \bigcup_{i \in I}\left\{r_{i}\right\}\right\rangle \nvdash \Delta$.

Now, the proof for $\langle W, R\rangle \nvdash \Delta$ ends with the following application of $(\mathbf{R e} \mathbf{3})$

$$
\frac{\left\langle W,\left\{r_{i_{1}}, \ldots, r_{i_{k-1}}\right\}\right\rangle \nvdash \Delta \quad\left\langle W,\left\{r_{i_{1}}, \ldots, r_{i_{k-1}}\right\}\right\rangle \nvdash \alpha_{i_{k}}}{\left\langle W,\left\{r_{i_{1}}, \ldots, r_{i_{k}}\right\}\right\rangle \nvdash \Delta}
$$

More generally, for all choices of $s, t$ with $\ell<s<t \leq k+1$ we use the (Re3)step

$$
\frac{\left\langle W,\left\{r_{i_{1}}, \ldots, r_{i_{s-1}}\right\}\right\rangle \nvdash \alpha_{i_{t}} \quad\left\langle W,\left\{r_{i_{1}}, \ldots, r_{i_{s-1}}\right\}\right\rangle \nvdash \alpha_{i_{s}}}{\left\langle W,\left\{r_{i_{1}}, \ldots, r_{i_{s}}\right\}\right\rangle \nvdash \alpha_{i_{t}}}
$$

where we set $\alpha_{k+1}=\bigvee \Delta$. After all these steps, it remains to derive the antisequents $\left\langle W,\left\{r_{i_{1}}, \ldots, r_{i_{\ell}}\right\}\right\rangle \nvdash \alpha_{i_{t}}$ for $\ell<t \leq k+1$. But for these we have already built the proofs $\Pi_{t}^{\prime}$. Therefore, we have constructed an $R C$-proof of $\langle W, R\rangle \nvdash \Delta$ which apart from the $A C$-proofs $\Pi_{t}^{\prime}$ uses only $\mathcal{O}\left(k^{2}\right)$ applications of (Re3) and (Re4). As each antisequent in the proof is of linear size, we obtain a polynomial-size $R C$-proof of $\langle W, R\rangle \nvdash \Delta$.

Let us remark that while the $R C$-proof of $\langle W, R\rangle \vdash \Delta$ in Fig. 3 is tree-like, this is not true for our dag-like $R C$-proof of $\langle W, R\rangle \nvdash \Delta$ constructed in the second part of the proof of Lemma 7.

\section{Proof Complexity of Credulous Default Reasoning}

Now we turn to the analysis of Bonatti and Olivetti's calculus for credulous default reasoning. An essential ingredient of the calculus are provability constraints which resemble a necessity modality. Provability constraints are of the 
form $\mathbf{L} \alpha$ or $\neg \mathbf{L} \alpha$ with $\alpha \in \mathcal{L}$. A set $E \subseteq \mathcal{L}$ satisfies a constraint $\mathbf{L} \alpha$ if $\alpha \in T h(E)$. Similarly, $E$ satisfies $\neg \mathbf{L} \alpha$ if $\alpha \notin T h(E)$.

We can now describe the calculus $B O_{\text {cred }}$ of Bonatti and Olivetti [4] for credulous default reasoning. A credulous default sequent is a 3-tuple $\langle\Sigma, \Gamma, \Delta\rangle$, denoted by $\Sigma ; \Gamma \sim \Delta$, where $\Gamma=\langle W, D\rangle$ is a default theory, $\Sigma$ is a set of provability constraints and $\Delta$ is a set of propositional sentences. Semantically, the sequent $\Sigma ; \Gamma \sim \Delta$ is true, if there exists a stable extension $E$ of $\Gamma$ which satisfies all of the constraints in $\Sigma$ and $\bigvee \Delta \in E$. The calculus $B O_{\text {cred }}$ uses such sequents and extends $L K, A C$, and $R C$ by the inference rules in Fig. 4 .

$$
\begin{aligned}
& \text { (cD1) } \frac{\Gamma \vdash \Delta}{; \Gamma \sim \Delta}\left(\Gamma \subseteq \mathcal{L}^{\text {res }}\right) \\
& \text { (cD2) } \frac{\Gamma \vdash \alpha \quad \Sigma ; \Gamma \sim \Delta}{\mathbf{L} \alpha, \Sigma ; \Gamma \sim \Delta}\left(\Gamma \subseteq \mathcal{L}^{\text {res }}\right) \quad(\text { cD3 }) \frac{\Gamma \nvdash \alpha \quad \Sigma ; \Gamma \sim \Delta}{\neg \mathbf{L} \alpha, \Sigma ; \Gamma \sim \Delta}\left(\Gamma \subseteq \mathcal{L}^{\text {res }}\right) \\
& \text { (cD4) } \frac{\mathbf{L} \neg \beta_{i}, \Sigma ; \Gamma \sim \Delta}{\Sigma ; \Gamma, \frac{\alpha: \beta_{1} \ldots \beta_{n}}{\gamma} \sim \Delta} \quad(\text { cD5 }) \frac{\neg \mathbf{L} \neg \beta_{1} \ldots \neg \mathbf{L} \neg \beta_{n}, \Sigma ; \Gamma, \frac{\alpha}{\gamma} \sim \Delta}{\Sigma ; \Gamma, \frac{\alpha: \beta_{1} \ldots \beta_{n}}{\gamma} \sim \Delta}
\end{aligned}
$$

Fig. 4. Inference rules for the credulous default calculus $B O_{\text {cred }}$.

For this calculus Bonatti and Olivetti [4] show the following:

Theorem 8 (Bonatti, Olivetti [4]). BO cred is sound and complete, i.e., a credulous default sequent is true if and only if it is derivable in $B O_{\text {cred }}$.

We now investigate lengths of proofs in $B O_{\text {cred }}$. Our next lemma shows that upper bounds on the proof size of $R C$ can be transferred to $B O_{\text {cred }}$.

Lemma 9. For any function $t(n)$, if $R C$ is $t(n)$-bounded, then $B O_{\text {cred }}$ is $p(n)$. $t(n)$-bounded for some polynomial $p$. The same relation holds for the number of steps in $R C$ and $B O_{\text {cred }}$.

Proof. Let $\Sigma ; \Gamma \sim \Delta$ be a true credulous default sequent. We will construct a $B O_{\text {cred }}$-derivation of $\Sigma ; \Gamma \sim \Delta$ starting from the bottom with the given sequent. Observe that we cannot use any of the rules (cD1) through (cD3) as long as $\Gamma$ contains proper defaults with nonempty justification. Thus we first have to reduce all defaults to residues plus some set of constraints using (cD4) or (cD5). As one of these rules has to be applied exactly once for each appearance of some default in $\Gamma$ we end up with $\Sigma^{\prime} ; \Gamma^{\prime} \sim \Delta$, where $\left|\Sigma^{\prime}\right|$ is polynomial in $|\Gamma \cup \Sigma|$ and $\Gamma^{\prime}$ is equal to $\Gamma$ on its propositional part and contains some of the corresponding residues instead of the defaults from $\Gamma$. From this point on we can only use rules (cD2) and (cD3) until we have eliminated all constraints and then finally apply rule (cD1) once. Thus, $B O_{\text {cred }}$-proofs look as shown in Fig. 5 where $R C$ indicates a derivation in the residual calculus and $\sigma$ is the remaining constraint from $\Sigma$ after applications of (cD2) or (cD3). Hence we obtain the bounds on $s_{B O_{\text {cred }}}$ and $t_{B O_{\text {cred }}}$. 


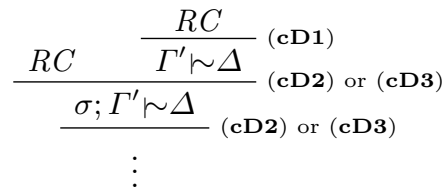

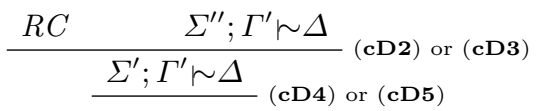

$$
\begin{aligned}
& \text { : } \\
& \Sigma ; \Gamma \sim \Delta
\end{aligned}
$$

Fig. 5. The structure of the $B O_{\text {cred }}$-proof in Lemma 9

Combining Lemmas 7 and 9 we obtain our main result in this section stating a tight connection between the proof complexity of $L K$ and $B O_{\text {cred }}$.

Theorem 10. There exist a polynomial $p$ and a constant $c$ such that $s_{L K}(n) \leq$ $s_{B O_{\text {cred }}}(n) \leq p(n) \cdot s_{L K}(c n)$ and $t_{L K}(n) \leq t_{B O_{\text {cred }}}(n) \leq p(n) \cdot t_{L K}(c n)$.

In the light of this result, proving either non-trivial lower or upper bounds to the proof size of $B O_{\text {cred }}$ seems very difficult - as such a result would mean a major breakthrough in propositional proof complexity (cf. [3,17]).

\subsection{On the Automatizability of $\mathrm{BO}_{\text {cred }}$}

Practitioners are not only interested in the size of a proof, but face the more complicated problem to actually construct a proof for a given instance. Of course, in the presence of super-polynomial lower bounds to the proof size this cannot be done in polynomial time. Thus, in proof search the best one can hope for is the following notion of automatizability:

Definition 11 (Bonet, Pitassi, Raz [6]). A proof system P for a language $L$ is automatizable if there exists a deterministic procedure that takes as input a string $x$ and outputs a P-proof of $x$ in time polynomial in the size of the shortest P-proof of $x$ if $x \in L$. If $x \notin L$, then the behaviour of the algorithm is unspecified.

For practical purposes automatizable systems would be very desirable. Searching for a proof we may not find the shortest one, but we are guaranteed to find one that is only polynomially longer. Unfortunately, for $B O_{\text {cred }}$ there are strong limitations towards this goal as our next result shows:

Theorem 12. $B O_{\text {cred }}$ is not automatizable unless factoring integers is possible in polynomial time.

Proof. First we observe that automatizability of $B O_{\text {cred }}$ implies automatizability of Frege systems. For this let $\varphi$ be a propositional tautology. By assumption, we can construct a $B O_{c r e d}$-proof of $\emptyset \sim \varphi$. This $B O_{c r e d}$-proof contains an $L K$-proof of $\emptyset \vdash \varphi$ by rule (cD1). As $L K$ is polynomially equivalent to Frege systems [17], we can construct from this $L K$-proof a Frege proof of $\varphi$ in polynomial time. By a result of Bonet, Pitassi, and Raz [6], Frege systems are not 
automatizable unless Blum integers can be factored in polynomial time (a Blum integer is the product of two primes which are both congruent 3 modulo 4 ).

\subsection{A General Construction of Proof Systems for Credulous Default Reasoning}

In this section we will explain a general method how to construct proof systems for credulous default reasoning. These proof systems arise from the canonical $\Sigma_{2}^{\mathrm{p}}$ algorithm for credulous default reasoning (Algorithm 1). Algorithm 1 first guesses a generating set $G_{\text {ext }}$ for a potential stable extension and then verifies by the stage construction from Theorem 1 that $G_{\text {ext }}$ indeed generates a stable extension which moreover contains the formula $\varphi$. Algorithm 1 is a $\Sigma_{2}^{\mathrm{p}}$ procedure, i.e., it can be executed by a nondeterministic polynomial-time Turing machine $M$ with access to a coNP-oracle. The nondeterminism solely lies in line 1 and the oracle queries are made in lines 6 and 11 to the coNP-complete problem of propositional implication $\operatorname{IMP}=\{\langle\Psi, \varphi\rangle \mid \Psi \subseteq \mathcal{L}, \varphi \in \mathcal{L}$, and $\Psi \models \varphi\}$.

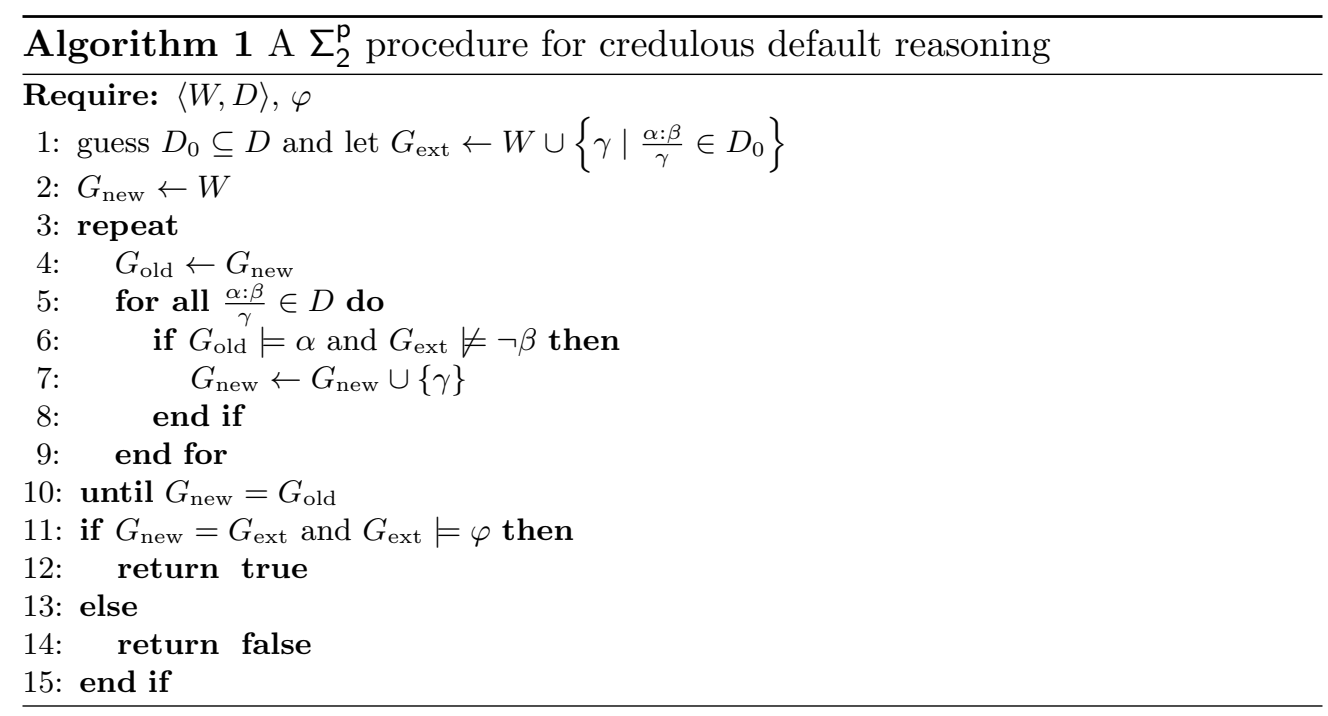

Algorithm 1 can be converted into a proof system for credulous default reasoning as follows. We fix a propositional proof system $P$ and define a proof system $\operatorname{Cred}(P)$ for credulous default reasoning where proofs are of the form

$$
\left\langle W, D, \varphi, c o m p, q_{1}, \ldots, q_{k}, a_{1}, \ldots, a_{k}\right\rangle \text {. }
$$

Here comp is a computation of $M$ on input $\langle W, D, \varphi\rangle$ and $q_{1}, \ldots, q_{k}$ are the queries to IMP during this computation. If the IMP-query $q_{i}=\left\langle\Psi_{i}, \varphi_{i}\right\rangle$ is answered positively, then $a_{i}$ is a $P$-proof of $\left(\bigwedge_{\psi \in \Psi_{i}} \psi\right) \rightarrow \varphi_{i}$, otherwise $a_{i}$ is an assignment falsifying this formula. For this proof system we obtain the following bounds:

Theorem 13. Let $P$ be a propositional proof system. Then $C r e d(P)$ is a proof system for credulous default reasoning with $s_{P}(n) \leq s_{C r e d(P)}(n) \leq \mathcal{O}\left(n^{2} s_{P}(n)\right)$. 
Proof. The first inequality holds because we can use $C r e d(P)$ to prove propositional tautologies $\varphi$ by choosing $W=D=\emptyset$.

For the second inequality, we observe that Algorithm 1 has quadratic running time. In particular, a computation of Algorithm 1 contains at most a quadratic number of queries to IMP. Each of these queries is of linear size because it only consists of formulae from the input. If the query is answered positively, then we have to supply a $P$-proof and there exists such a $P$-proof of size $\leq s_{P}(n)$. For a negative answer we just include an assignment of linear size. This yields $s_{C r e d(P)}(n) \leq \mathcal{O}\left(n^{2} s_{P}(n)\right)$.

Theorem 13 tells us that proving lower bounds for proof systems for credulous default reasoning is more or less the same as proving lower bounds to propositional proof systems. In particular, we get:

Corollary 14. There exists a polynomially bounded proof system for credulous default reasoning if and only if there exists a polynomially bounded propositional proof system.

\section{Lower Bounds for Skeptical Default Reasoning}

Bonatti and Olivetti [4] introduce two calculi for skeptical default reasoning. As before, objects are sequents of the form $\Sigma ; \Gamma \sim \Delta$, where $\Sigma$ is a set of constraints, $\Gamma$ is a propositional default theory, and $\Delta$ is a set of propositional formulae. But now, the sequent $\Sigma ; \Gamma \sim \Delta$ is true, if $\bigvee \Delta$ holds in all extensions of $\Gamma$ satisfying the constraints in $\Sigma$.

The first calculus $B O_{\text {skep }}$ consists of the defining axioms of $L K$ and $A C$, the inference rules of $L K, A C, R C$, and the rules from Fig. 6. Bonatti and

$$
\begin{gathered}
(\mathbf{s D 1}) \frac{\Gamma \vdash \Delta}{\Sigma ; \Gamma \sim \Delta}\left(\Gamma \subseteq \mathcal{L}^{\text {res }}\right) \\
(\mathbf{s D 2}) \frac{\Gamma \vdash \alpha}{\neg \mathbf{L} \alpha, \Sigma ; \Gamma \sim \Delta}\left(\Gamma \subseteq \mathcal{L}^{\text {res }}\right) \\
(\mathbf{s D 4}) \frac{\neg \mathbf{L} \neg \beta_{1}, \ldots, \neg \mathbf{L} \neg \beta_{n}, \Sigma ; \Gamma, \frac{\alpha}{\gamma} \sim \Delta \quad(\mathbf{s D 3}) \frac{\Gamma \nvdash \alpha}{\mathbf{L} \alpha, \Sigma ; \Gamma \sim \Delta}\left(\Gamma \subseteq \mathcal{L}^{\text {res }}\right)}{\Sigma ; \Gamma, \frac{\alpha: \beta_{1} \ldots \beta_{n}}{\gamma} \sim \Delta ; \Gamma \sim \Delta \quad \ldots \quad \mathbf{L} \neg \beta_{n}, \Sigma ; \Gamma \sim \Delta}
\end{gathered}
$$

Fig. 6. Inference rules for the skeptical default calculus $B O_{\text {skep }}$.

Olivetti show that each true sequent is derivable in $B O_{\text {skep }}$, i.e., the calculus is sound and complete. However, they already remark that proofs in $B O_{\text {skep }}$ are of exponential size in the number of default rules in the sequent. This is due to the residual rules for they cannot be applied unless all defaults with nonempty justifications have been eliminated using rule (sD4).

To get more concise proofs, Bonatti and Olivetti [4] suggest an enhanced calculus $B O_{\text {skep }}^{\prime}$ where the rules $(\mathbf{s D 1})$ to $(\mathbf{s D 3})$ are replaced by rules $\left(\mathbf{s D 1} \mathbf{1}^{\prime}\right)$ to 
$\left(\mathbf{s D 3}^{\prime}\right)$ and rule (sD4) is kept (see Fig. 7). Bonatti and Olivetti prove soundness and completeness for $B O_{\text {skep }}^{\prime}$. Moreover, they show that $B O_{\text {skep }}^{\prime}$ is exponentially separated from $B O_{\text {skep }}$, i.e., there exist sequents $\left(S_{n}\right)_{n \geq 1}$ which require exponential-size proofs in $B O_{\text {skep }}$ but have linear-size derivations in $B O_{\text {skep }}^{\prime}$. In

$$
\begin{aligned}
& \text { (sD1') } \frac{\Sigma^{\prime}, \Gamma^{\prime} \vdash \Delta}{\Sigma ; \Gamma \sim \Delta} \quad\left(\mathbf{s D 2}^{\prime}\right) \frac{\Sigma ; \Gamma \sim \alpha}{\neg \mathbf{L} \alpha, \Sigma ; \Gamma \sim \Delta} \quad\left(\mathbf{s D 3}^{\prime}\right) \frac{\Gamma^{\prime \prime} \nvdash \alpha}{\mathbf{L} \alpha, \Sigma ; \Gamma \sim \Delta} \\
& \text { (sD4) } \frac{\neg \mathbf{L} \neg \beta_{1}, \ldots, \neg \mathbf{L} \neg \beta_{n}, \Sigma ; \Gamma, \frac{\alpha}{\gamma} \sim \Delta \quad \mathbf{L} \neg \beta_{1}, \Sigma ; \Gamma \sim \Delta \quad \ldots \quad \mathbf{L} \neg \beta_{n}, \Sigma ; \Gamma \sim \Delta}{\Sigma ; \Gamma, \frac{\alpha: \beta_{1} \ldots \beta_{n}}{\gamma} \sim \Delta} \\
& \text { where } \Sigma^{\prime} \subseteq\{\alpha \mid \mathbf{L} \alpha \in \Sigma\}, \Gamma^{\prime} \subseteq \Gamma \cap \mathcal{L}^{\text {res }}, \text { and } \Gamma^{\prime \prime}=(\Gamma \cap \mathcal{L}) \cup\left\{\frac{p(\delta)}{c(\delta)} \mid \delta \in \Gamma\right\} .
\end{aligned}
$$

Fig. 7. Inference rules for the enhanced skeptical default calculus $B O_{\text {skep }}^{\prime}$.

our next result we will show an exponential lower bound to the proof length (and therefore also to the proof size) in the enhanced skeptical calculus $B O_{\text {skep }}^{\prime}$.

Theorem 15. The calculus $B O_{\text {skep }}^{\prime}$ has exponential lower bounds to the lengths of proofs. More precisely, there exist sequents $S_{n}$ of size $\mathcal{O}(n)$ such that every $B O_{\text {skep }}^{\prime}$-proof of $S_{n}$ uses $2^{\Omega(n)}$ steps. Therefore, $s_{B O_{\text {skep }}^{\prime}}(n), t_{B O_{\text {skep }}^{\prime}}(n) \in 2^{\Omega(n)}$.

Proof. (Sketch) We construct a sequence $\left(S_{n}\right)_{n \geq 1}=\left(\Sigma_{n} ; \Gamma_{n} \sim \psi_{n}\right)_{n \geq 1}$ such that for some constant $c$, every $B O_{\text {skep }}^{\prime}$-proof of $S_{n}$ has length at least $2^{\Omega(n)}$. We choose $\Sigma_{n}=\emptyset, \psi_{n}=A_{2 n}$, and $\Gamma_{n}=\left\langle\emptyset, D_{2 n}\right\rangle$, where $D_{2 n}$ consists of the defaults listed in Fig. 8. The default theory $\Gamma_{n}$ possesses $2^{n+1}$ stable extensions. Observe that each of these contains $A_{2 n}$, but that each pair of stable extensions differs in truth assigned to the propositional variables $A_{0}, \ldots, A_{n}$. We claim that every proof of $S_{n}$ has exponential length in $n$. More precisely, we will show that rule (sD4) has to be applied an exponential number of times.

We point out that our argument does not only work against tree-like proofs, but also rules out the possibility of sub-exponential dag-like derivations for $D_{2 n} \sim A_{2 n}$. The lower bound is obtained from the fact that to derive $A_{2 n}$, we have to derive $A_{i}$ and $\neg A_{i}$ for each $n<i<2 n$, each of which can only be achieved from ancestors with mutually different proof constraints. This, by definition of $B O_{\text {skep }}$, leads to mutually disjoint sets of ancestor sequents.

\section{Conclusion}

In this paper we have shown that with respect to lengths of proofs, proof systems for credulous default reasoning and for propositional logic are very close to each other. Although deciding credulous default sequents is presumably harder than deciding tautologies (the former is $\Sigma_{2}^{\mathrm{p}}$-complete [13], while the latter is complete for coNP), the difference disappears when we want to prove these objects (Sect. 4.2). 


$$
\begin{aligned}
& \frac{: A_{0}}{A_{0}} \quad \frac{: \neg A_{0}}{\neg A_{0}} \\
& \frac{A_{0}: A_{1}}{A_{1}} \quad \frac{\neg A_{0}: A_{1}}{A_{1}} \quad \frac{A_{0}: \neg A_{1}}{\neg A_{1}} \quad \neg \quad \frac{\neg A_{0}: \neg A_{1}}{\neg A_{1}} \\
& \vdots \\
& \frac{A_{n-1}: A_{n}}{A_{n}} \quad \frac{\neg A_{n-1}: A_{n}}{A_{n}} \quad \frac{A_{n-1}: \neg A_{n}}{\neg A_{n}} \quad \neg \quad \frac{\neg A_{n-1}: \neg A_{n}}{\neg A_{n}} \\
& \frac{A_{n}: A_{n-1}}{A_{n+1}} \quad \frac{\neg A_{n}: A_{n-1}}{A_{n+1}} \quad \frac{A_{n}: \neg A_{n-1}}{\neg A_{n+1}} \quad \neg \quad \frac{\neg A_{n}: \neg A_{n-1}}{\neg A_{n+1}} \\
& \frac{A_{2 n-2}: A_{1}}{A_{2 n-1}} \frac{\neg A_{2 n-2}: A_{1}}{A_{2 n-1}} \quad \frac{A_{2 n-2}: \neg A_{1}}{\neg A_{2 n-1}} \quad \neg \quad \frac{\neg A_{2 n-2}: \neg A_{1}}{\neg A_{2 n-1}} \\
& \frac{A_{2 n-1}: A_{0}}{A_{2 n}} \quad \frac{\neg A_{2 n-1}: A_{0}}{A_{2 n}} \quad \frac{A_{2 n-1}: \neg A_{0}}{A_{2 n}} \quad \neg \quad \frac{\neg 2 n-1}{A_{2 n}} \quad \neg A_{0}
\end{aligned}
$$

Fig. 8. The defaults in $D_{2 n}$ in the proof of Theorem 15 .

For skeptical reasoning this is less clear. While skeptical default reasoning has polynomially bounded proof systems if and only if this holds for TAUT, we leave open whether this equivalence extends to other bounds. However, in the light of our exponential lower bound for $B O_{\text {skep }}^{\prime}$ (Theorem 15), searching for natural proof systems for skeptical default reasoning with more concise proofs will be a rewarding task for future research.

In this direction Bonatti and Olivetti [4] themselves introduced two rules to supplement their enhanced calculus. These are the cut rule

$$
\frac{\Sigma ; \Gamma \sim \alpha \quad \Sigma ; \Gamma, \alpha \sim \Delta}{\Sigma ; \Gamma \sim \Delta}(\mathbf{C u t})
$$

and the following version of the rule (sD4)

$$
\frac{\Sigma_{0}, \Sigma ; \Gamma, \frac{\alpha}{\gamma} \sim \Delta \quad \Sigma_{1}, \Sigma ; \Gamma \sim \Delta \quad \ldots \quad \Sigma_{n}, \Sigma ; \Gamma \sim \Delta}{\Sigma ; \Gamma, \frac{\alpha: \beta_{1} \ldots \beta_{n}}{\gamma} \sim \Delta}\left(\mathbf{s D 4}^{\prime}\right)
$$

where $\Sigma_{i}=\mathbf{L} \neg \beta_{\pi(i)}, \neg \mathbf{L} \neg \beta_{\pi(i+1)}, \ldots, \neg \mathbf{L} \neg \beta_{\pi(n)}$ for an arbitrary permutation $\pi$ of $\{1, \ldots, n\}$. While it is not hard to see that our lower bound in Theorem 15 still remains true if we add $\left(\mathbf{s} \mathbf{D 4}^{\prime}\right)$ to $B O_{\text {skep }}^{\prime}$, we leave open the problem to show super-polynomial lower bounds in the presence of the cut rule.

\section{Acknowledgement}

The first author wishes to thank Neil Thapen for interesting discussions on the topic of this paper during a research visit to Prague. 


\section{References}

1. G. Amati, L. C. Aiello, D. M. Gabbay, and F. Pirri. A proof theoretical approach to default reasoning I: Tableaux for default logic. Journal of Logic and Computation, 6(2):205-231, 1996.

2. G. Antoniou. A tutorial on default logics. ACM Comput. Surv., 31(4):337-359, 1999.

3. P. A. Bonatti. A Gentzen system for non-theorems. Technical Report CD/TR 93/52, Christian Doppler Labor für Expertensysteme, 1993.

4. P. A. Bonatti and N. Olivetti. Sequent calculi for propositional nonmonotonic logics. ACM Transactions on Computational Logic, 3(2):226-278, 2002.

5. M. L. Bonet, S. R. Buss, and T. Pitassi. Are there hard examples for Frege systems? In P. Clote and J. Remmel, editors, Feasible Mathematics II, pages 30-56. Birkhäuser, 1995.

6. M. L. Bonet, T. Pitassi, and R. Raz. On interpolation and automatization for Frege systems. SIAM Journal on Computing, 29(6):1939-1967, 2000.

7. M. Cadoli and M. Schaerf. A survey of complexity results for nonmonotonic logics. Journal of Logic Programming, 17(2/3\&4):127-160, 1993.

8. S. A. Cook and R. A. Reckhow. The relative efficiency of propositional proof systems. The Journal of Symbolic Logic, 44(1):36-50, 1979.

9. J. Dix, U. Furbach, and I. Niemelä. Nonmonotonic reasoning: Towards efficient calculi and implementations. In Handbook of Automated Reasoning, pages 1241-1354. Elsevier and MIT Press, 2001.

10. U. Egly and H. Tompits. Proof-complexity results for nonmonotonic reasoning. ACM Transactions on Computational Logic, 2(3):340-387, 2001.

11. D. Gabbay. Theoretical foundations of non-monotonic reasoning in expert systems. In Logics and Models of Concurrent Systems, pages 439-457. Springer-Verlag, Berlin Heidelberg, 1985.

12. G. Gentzen. Untersuchungen über das logische Schließen. Mathematische Zeitschrift, 39:68-131, 1935.

13. G. Gottlob. Complexity results for nonmonotonic logics. Journal of Logic and Computation, 2(3):397-425, 1992.

14. P. Hrubeš. On lengths of proofs in non-classical logics. Annals of Pure and Applied Logic, 157(2-3):194-205, 2009.

15. E. Jeřábek. Frege systems for extensible modal logics. Annals of Pure and Applied Logic, 142:366-379, 2006.

16. E. Jeřábek. Substitution Frege and extended Frege proof systems in non-classical logics. Annals of Pure and Applied Logic, 159(1-2):1-48, 2009.

17. J. Krajíček. Bounded Arithmetic, Propositional Logic, and Complexity Theory, volume 60 of Encyclopedia of Mathematics and Its Applications. Cambridge University Press, Cambridge, 1995.

18. S. Kraus, D. J. Lehmann, and M. Magidor. Nonmonotonic reasoning, preferential models and cumulative logics. Artificial Intelligence, 44(1-2):167-207, 1990.

19. D. Makinson. General theory of cumulative inference. In Proc. 2nd International Workshop on Non-Monotonic Reasoning, pages 1-18. Springer-Verlag, Berlin Heidelberg, 1989.

20. V. W. Marek and M. Truszczyński. Nonmonotonic Logics-Context-Dependent Reasoning. Springer-Verlag, Berlin Heidelberg, 1993.

21. R. Reiter. A logic for default reasoning. Artificial Intelligence, 13:81-132, 1980.

22. V. Risch and C. Schwind. Tableaux-based characterization and theorem proving for default logic. Journal of Automated Reasoning, 13(2):223-242, 1994.

23. M. L. Tiomkin. Proving unprovability. In Proc. 3rd Annual Symposium on Logic in Computer Science, pages 22-26, 1988. 